\title{
Erhöhtes Risiko für ältere Menschen und junge Frauen
}

_ Dass ältere Menschen, die sich falsch ernähren, zu Eisenmangelanämien neigen, ist nachvollziehbar. Eine oft unterschätzte Risikogruppe für Eisenmangel sind aber auch junge Frauen, die sich $\mathrm{z}$. B. bewusst gegen den Verzehr von Fleisch entschieden haben und die sich damit eisenreduziert ernähren [1].

Ein Eisenmangel kann sich in einem fortgeschrittenen Stadium in einer Anämie äußern (Hämoglobin[Hb]-Wert $<12 \mathrm{~g} / \mathrm{dl}$ ). Aber auch bereits ein prälatenter Eisenmangel (normaler Hb-Wert, Ferritinspiegel $<50 \mu \mathrm{g} / \mathrm{l})$ kann zu therapiebedürftigen Beschwerden wie chronischer Müdigkeit, Antriebslosigkeit, gestörter Konzentrationsfähigkeit, reduzierter Muskelleistung und Leistungsschwäche (Fatigue) führen.
Dass eine orale Eisensubstitution sogar bei einem $\mathrm{Hb}$ im Normalbereich die Fatigue-Symptomatik deutlich verbessern kann, zeigte eine Multicenterstudie mit 198 prämenopausalen Frauen, die einen Eisenmangel ohne Anämie hatten und unter Fatigue litten. Unter einer zwölfwöchigen Therapie mit 1 x $80 \mathrm{mg} / \mathrm{d}$ Depot-Eisen-II-sulfat (Tardyferon ${ }^{\circledR}$ ) stiegen zum einen die Hämoglobin-, Ferritinund Hämatokritwerte an. Zum anderen wurde die Fatigue-Symptomatik im Vergleich zur Placebogruppe deutlich verbessert [2].

\section{Entscheidend: das richtige Eisenpräparat}

Wichtig für einen derartigen Therapieerfolg sind neben der zugeführten Ei- senmenge die Resorbierbarkeit und die Verträglichkeit des Eisenpräparates. Bei Tardyferon ${ }^{\circledast}$ wurde eine Formulierung entwickelt, welche das Eisen im Darm verzögert freisetzt. Dies ermöglicht eine bessere Eisenaufnahme durch die enteralen Transportsysteme. In einem Review von 111 Studien mit 10.695 Patienten konnte gezeigt werden, dass unter der Therapie mit DepotEisen-II-sulfat im Vergleich zu anderen oralen Eisenpräparaten die wenigsten gastrointestinalen Begleiteffekte auftreten.

Red.

1. Bitzer et al. Gynecol Endocrinol.2014;30:542-8

2. Vaucher et al. CMAJ. 2012; 184:1247-54

3. Cancelo-Hidalgo et al. Curr Med Res Opin. 2013 29:291-301

\section{Asthma bronchiale}

\section{So schützen Sie Ihre Allergiker auch im Herbst}

_ IgE spielt eine zentrale Rolle bei allergischen Entzündungsreaktionen. Durch seine Bindung an dendritische Zellen erleichtert es die Allergenpräsentation in vivo. Zudem wird bei seiner Anwesenheit die Interferon- $\alpha$-Bildung von dendritischen Zellen gehemmt, sodass die virale Abwehr beeinträchtigt wird. Dies erklärt die saisonale Häufung von infektbedingten Asthmaexazerbationen im Herbst.

Omalizumab blockiert IgE und stoppt so die Entzündungsreaktionen. In einer Analyse von acht Pilotstudien zeigte sich unter Omalizumab eine konsistente Reduktion von Asthmaexazerbationen. Einen Beweis für die Wirksamkeit dieses Behandlungskonzepts lieferte die PROSE-Studie, in der Kinder mit persistierendem schwerem allergischem Asth- ma zunächst von November bis Juli 4-9 Monate lang behandelt wurden, bis ihr Asthma kontrolliert war. Im Anschluss erhielten die Kinder 4-6 Wochen vor Schulbeginn entweder Omalizumab oder vermehrt inhalative Steroide (ICS).

Durch Omalizumab wurden die Exazerbationen vs. Placebo halbiert, ICS hatten dagegen keine präventive Wirkung. „Wir erklären uns diese Wirkung so, dass Omalizumab dendritische Zellen wieder in die Lage versetzt, Interferon- $\alpha$ zu produzieren und so virale Infektionen abzuwehren“, erklärte Prof. William Busse, Madison (WI/USA).

Inzwischen haben mehrere Studien gezeigt, dass Omalizumab Asthmaexazerbationen sowie den Einsatz von Kortikosteroiden verringern kann und die Lebensqualität bei Kindern und Erwach- senen mit schwerem allergischem Asthma verbessert.

\section{Dr. Susanne Kammerer}

- Satellitensymposium „50years of IgE: What we've learnt and envisioning the future", Kongress der European Academy of Allergy and Clinical Immunology; Wien, Juni 2016 (Veranstalter: Novartis)

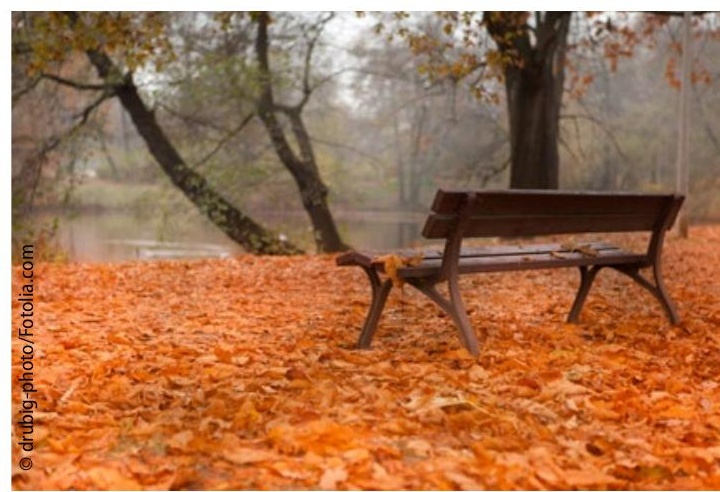

Auch im Herbst draußen sein können ... 